\title{
Community drug distributors for mass drug administration in neglected tropical disease programmes: systematic review and analysis of policy documents
}

\author{
Cara L Macfarlane ${ }^{1}$, Laura \\ Dean², Rachael Thomson ${ }^{3}$, \\ Paul Garner ${ }^{1}$ \\ ${ }^{1}$ Centre for Evidence Synthesis in Global \\ Health, Department of Clinical Sciences, \\ Liverpool School of Tropical Medicine, \\ Liverpool, UK \\ ${ }^{2}$ Department of International Public Health, \\ Liverpool School of Tropical Medicine, \\ Liverpool, UK \\ ${ }^{3}$ Department for Tropical Disease Biology, \\ Liverpool School of Tropical Medicine, \\ Liverpool, UK
}

Background Mass drug administration (MDA) programmes for neglected tropical diseases (NTDs) depend on voluntary community drug distributors (CDDs) to deliver drugs, and these volunteer schemes need regular training and supervision. NTD policy now includes integration of multiple disease programmes, but we are unsure if there is clarity in what is currently expected of CDDs and how they are managed. We therefore analysed World Health Organization (WHO) policy, strategy and implementation guidance, and select national NTD programme implementation plans.

Methods Included are a) WHO global and WHO-Regional Office for Africa guidelines, strategies, operational manuals and meeting reports published between January 2007 to February 2018 that included policy and plans for CDDs; and b) national NTD programme master plans for Cameroon, Ghana, Liberia and Nigeria. For both review components, we examined the CDD responsibilities through a framework developed iteratively against the documents and prepared a narrative synthesis.

Results Twenty WHO policy documents met the inclusion criteria. In the twelve global and eight regional documents, the CDD role was not explicitly or comprehensively defined. Three documents mentioned CDDs will distribute drugs; some mentioned health promotion, data handling and engagement in clinical care. Four WHO documents noted a need for CDD training or management, eight detailed some aspect of this, and one regional document provided a comprehensive overview. In the national plans, additional responsibilities included case management in two countries and transmission control in two countries. Every plan included training and supervision, but this was not always explicit, and details of the purpose and frequency varied. In all national plans, CDD motivation was identified as a challenge but not comprehensively addressed, although one document mentioned provision of bicycles.

Conclusions WHO and national policies and plans assume CDDs will implement NTD programmes. However, there is almost no clear delineation of responsibilities, nor is there up-to-date practical guidance to guide managers. This ambiguity, in relation to the lack of explicit policies or programmatic guidance, probably impairs the effectiveness of NTD programmes.

\section{This project}

We were part of development teams to supporting sustainable scaling up of mass drug administration (MDA) campaigns in Cameroon, Liberia, Ghana and Nigeria (http://countdown.lstmed.ac.uk/), and were asked to exam- 
ine the evidence around volunteer community drug distributors (CDDs) in neglected tropical disease (NTD) programmes.

In our initial appraisal, we identified clear benchmarks in relation to a World Health Organization (WHO) affiliated training document for CDDs published in 1998 [1] but found more recent policies relating to CDDs were not as well-defined. As this cadre appears to be central to global programmes distributing freely donated drugs [2], we wondered how clear are policies and procedures set by WHO or national programmes to assure their distribution by CDDs? What are the explicit responsibilities of CDDs in contemporary MDA programmes for NTDs in Africa?

We address these questions in this analysis, but we first provide a brief overview of the history of NTD policy from 1995 to present day. We then systematically sought and analysed the CDDs role as presented in a) global and regional policy documents for Africa; and b) in select national NTD programme master plans.

\section{Historical overview}

The WHO currently recognises 20 communicable diseases prevalent in tropical and subtropical conditions worldwide [3], collectively called neglected tropical diseases or NTDs. NTDs are associated with poverty, and primarily impact populations in low-income and middle-income countries with limited access to under-resourced health services [4]. NTDs are a diverse group of infections and conditions that adversely impact mental health [5], and cause considerable morbidity [6], social stigma [7] and death [8]. The African Region alone suffers around $40 \%$ of the global burden of NTDs [9].

Five NTDs - onchocerciasis, lymphatic filariasis, soil-transmitted helminthiasis (STHs), schistosomiasis and trachoma - can be managed through preventive chemotherapy (PC) and are termed PC-NTDs. When given annually or semi-annually to the entire population at risk (MDA) or specific at-risk groups (targeted treatment), PC can treat individuals for the infection, prevent new morbidity developing over time and reduce or interrupt disease transmission [10]. Of the 47 countries in the WHO African Region, 44 are endemic for one or more NTDs that are amenable to PC, and 17 countries are co-endemic for five of these diseases [2].

Control programmes require repeated rounds of drug delivery over several years to endemic areas, with high therapeutic and geographic coverage, to meet the public health targets for the PC-NTDs. Community-based delivery of PC is dependent on a cadre of health volunteers, termed community drug distributors or CDDs, to distribute treatment within their communities, particularly in hard to reach and remote areas [2].

\section{Origins}

The community-directed treatment (ComDT) approach engaging volunteer CDDs for drug distribution was developed by the WHO and Special Programme for Research and Training in Tropical Diseases (TDR), and subsequently adopted by the African Programme for Onchocerciasis Control (APOC) in the late 1990s after a multi-country study showed that use of CDDs was a feasible and effective approach to deliver ivermectin for onchocerciasis [11]. This strategy became known as community-directed treatment with ivermectin (CDTI). CDDs were related but seen as separate to community health workers (CHWs), who often had a broader role in communities with health promotion and advice and could be paid for their services.

The "Practical Guide for Trainers of Community-Directed Distributors" was published by APOC in 1998, and CDDs were described as "villagers who have been selected by their communities to carry out the task of distributing ivermectin to other members of their community" [1]. This document was extensive and included detailed guidance on how CDDs should be selected, how to conduct their training and tasks for the trainee CDD post-training. Responsibilities of CDDs included conducting a village census to determine the quantity of drugs required, keeping a drug inventory, keeping records and liaising with health workers, providing the correct drug dosage, excluding people ineligible for treatment (pregnant and breastfeeding women, sick people) and referring people experiencing adverse reactions to a health facility [1]. The community was responsible for incentivising CDDs, and sustainability of the programme stemmed from greater ownership and empowering communities to have control over their own health.

\section{Integration}

Following the initial success of the CDTI strategy, which included 20 million people being treated annually and 67000 CDDs trained by 2001 [12], the CDTI network was soon used to deliver additional com- 
munity health services $[12,13]$ and explored by other NTD programmes, including lymphatic filariasis [14] and schistosomiasis and STHs [15].

In 2003 and 2005, the WHO held international workshops that coined the collective term "neglected tropical diseases" and started the transition from vertical disease-centred approaches to an integrated community-centric approach $[16,17]$. The narrative changed to the promotion of integrated NTD programmes, where many of the PC-NTDs have overlapping geographical distributions and treatment regimens [10,18-21]. In 2006, the WHO launched a new drug strategy for integrated PC for helminthiasis [10], and the United States Agency for International Development (USAID) established the NTD Control Program (NTDCP) to test integration on a large scale and facilitate expansion of integrated treatment in select national programmes [22,23].

The first "Global Plan to combat Neglected Tropical Diseases (2008-2015)", published by the WHO in 2007 , then endorsed integrating NTD control programmes worldwide with co-implementation of PC as a 'drug package' [24]. The NTD Roadmap was later published to guide implementation of the policies and strategies outlined in the global NTD plan, and quickly followed by support from the London Declaration on NTDs [25].

In the WHO African Region, integrated PC is currently a priority intervention in the "Regional Strategy on NTDs in the WHO African Region (2014-2020)" and includes the co-implementation of community-directed interventions (CDI) [26]. Country-specific strategies for integrated NTD control and elimination are stipulated in the multi-year master plan for national NTD programmes, in accordance with WHO guidelines [27].

\section{Objective}

To delineate the role of CDDs in contemporary MDA programmes for NTDs in the African Region, as defined in global and regional policy documents; and as outlined in a selection of national NTD programme master plans.

\section{METHODS}

\section{Inclusion criteria}

For the policy documents, we included documents from WHO global programmes and documents from the WHO Regional Office for Africa (WHO-AFRO). We sought WHO guidelines, strategies, operational manuals and meeting reports published between January 2007 to February 2018 that included policy and plans for CDDs in PC-NTD programmes. Reports of meetings were included if there were recommendations or action points relating to CDDs. We took 2007 as the starting point given the establishment of worldwide NTD policy by the WHO in this year, which included the first global partner's meeting on NTDs [28] and publication of the global NTD plan [24]. This set the international agenda for NTD prevention, control, elimination or eradication, and we would expect any resultant changes in the expectations of CDDs and their management to be outlined clearly in the policies and plans that followed.

Exclusion criteria consisted of documents described as "working drafts", reports, documents that only referred to CDDs and related terms in the introductory sections or in a "situation analysis", "update" or "progress report", and documents that referred only to CDI or CDTI without clear reference to CDD involvement.

For the second objective, we sought the NTD programme master plans from Cameroon, Ghana, Liberia, and Nigeria. These countries were selected as they all receive financial and technical support from the COUNTDOWN consortium (a Department for International Development (DFID)-funded project). These countries use the standard NTD programme template and were selected to be part of the COUNTDOWN consortium as they are all in different phases of NTD control (https://countdown. lstmed.ac.uk/).

\section{Definition of a community drug distributor}

The CDD cadre has various labels and expectations, and so our definition is: "a volunteer who is selected by the community to distribute drugs for diseases targeted by NTD programmes". This includes volunteers that participate in MDA to all members of a community and volunteers involved in targeted treat- 
ment of specific at-risk groups in the community [10]. Within the context of this review, we use a slightly modified definition of the CHW described by Lewin et al [29] to include any health volunteer who:

- performs functions related to drug distribution to prevent selected NTDs;

- is trained in some way in the context of the intervention; but

- has no formal professional or paraprofessional certificate or tertiary education degree.

This definition of the CDD cadre excludes teachers, frontline health workers and other individuals who may be involved in drug distribution in conjunction with their salaried employment.

\section{Policy documents}

\section{Search}

We attempted to identify all relevant WHO policy documents published between January 2007 to February 2018 by searching https://www.who.int with "Global" as the regional site (English only), the WHO-AFRO Library and the WHO IRIS AFRO collections (no language restrictions) using the search terms and subjects listed in Table S1 in Online Supplementary Document. We also searched the APOC website and the Expanded Special Project for Elimination of Neglected Tropical Diseases (ESPEN) portal resources for publications. We noted any documents that are currently in preparation or documents we are aware of but unable to obtain a copy. We engaged with experts from the research, advocacy and policy sectors in the field of NTDs to ensure inclusivity of all relevant policy documents.

\section{Selection}

Records identified from the search strategy were combined and titles screened to remove duplicate and irrelevant records. Records that were clearly not policy documents, such as WHO Weekly Epidemiological Reports, brochures and newsletters, were excluded.

\section{Data identification}

Full-text copies of all potentially relevant policy documents were obtained and assessed by one author based on the inclusion criteria using NVivo 11 Software (QSR International, Melbourne, Australia). Text search was used to identify sought-after text, such as "distributor", in each document, applying the "with stemmed words" setting to identify similar text (eg, distributors). The list of text terms is provided in Table S1 in the Online Supplementary Document. When it was unclear, a second author was consulted. Ineligible documents and the reasons for their exclusion were listed in a "characteristics of excluded documents" table.

\section{Data extraction}

Pre-tested data extraction forms were used to manage the data. We extracted information on the type of document, the publication date, the scope of the document (global or regional policy), the disease(s) covered and statements relevant to the drug distributor identified through the NVivo text search. For each document, the statements were organised into groups, including: 1 . assertions about their role; 2 . recommendations for their role; 3. reference to their training and management; and 4. other comments about CDDs.

\section{Data analysis}

We prepared a narrative synthesis of the role of CDDs as is documented in global and regional documents. We examined the documents and the data and then used this to inform the categories. Documents were assessed using four broad categories that were subdivided to reflect the description of the CDDs role, the strength of the policy, and the level of detail provided:

1. Specifying aspects of the CDDs role. The main options were drug distribution, data collection, health promotion, and a role in clinical care.

2. Recommendations for the CDDs role. Options included: none, an option with caveats, an option, and a formal recommendation.

3. Strategies for training and managing CDDs. Options included: no strategies, notes need, comment on aspects, and clearly and comprehensively outlined.

4. Other comments about CDDs. 
We considered "specifying aspects of the CDDs role" as a directive statement about an activity CDDs are or will be involved in, while "other comments about CDDs" were more general statements that included other relevant information about CDDs but were not instructional.

\section{National programme master plans}

The national NTD programme master plans follow the structure recommended in the WHO "Guide for preparing a master plan for national NTD programmes in the African region" (Figure 1) [27], and include the national objectives, goals and a 3-5 year strategy to guide programme planning and implementation. We obtained the most recent national NTD master plan from Cameroon, Liberia, Nigeria and Ghana through the COUNTDOWN consortium and the ESPEN portal resources.

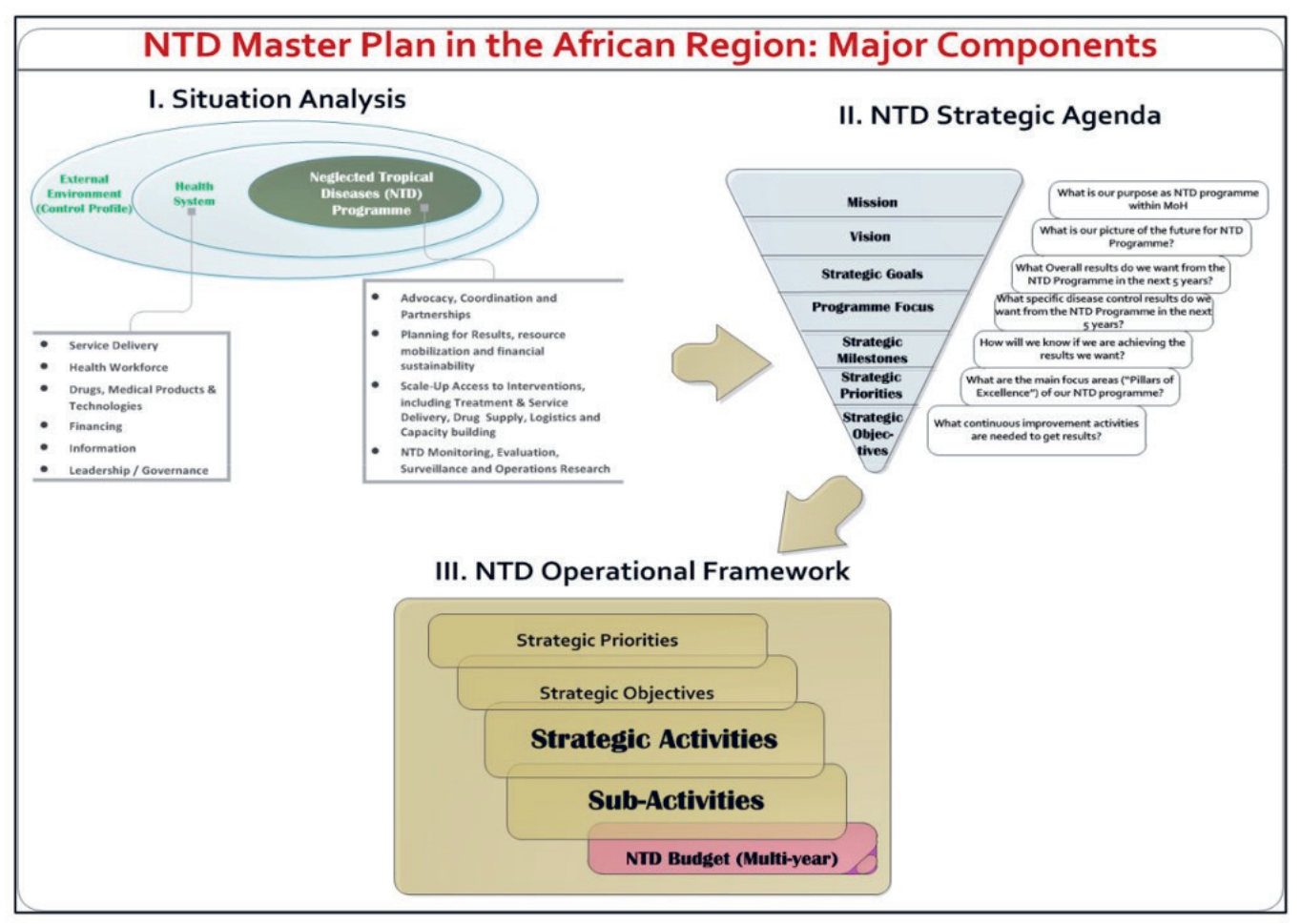

Figure 1. Major components of neglected tropical disease programme master plans for the African Region outlined by the World Health Organization [27].

\section{Data extraction}

Data extraction recorded the date of publication the date of publication and period covered by the plan, the disease(s) covered and information relevant to the drug distributor, including the diseases requiring drug delivery by CDDs, any other activities CDDs are implicated in, details of training and supervision, and whether incentives are included. Prior to using text search to extract relevant data, we scoped the master plan glossaries and introductory "situation analysis" sections to identify any other country-specific terms for CDDs. We then conducted the same text search as was used for the global and regional documents, with the addition of any country-specific terms for CDDs and the terms supervis* and train*.

\section{Data analysis}

A narrative synthesis was prepared to document the intended role of CDDs as it is outlined in the NTD programme plans of Cameroon, Ghana, Liberia and Nigeria. Statements in the plans regarding CDDs were analysed by compiling information relating to:

1. Anticipated role of CDDs, plus any additional activities of volunteers mentioned in the "situation analysis".

2. Strategies for training and management.

3. Motivation and incentives (motivation is acknowledged, and incentives included). 


\section{RESULTS}

\section{Policy documents}

Twenty documents in 24 records met the inclusion criteria (Figure 2). One document, the regional strategy on NTDs in the WHO African Region [26], had three related records [26,30-32] but there was no difference in relation to content on CDDs, so we report this as one document [26]. Another document [33] had one related record [34], which was a shorter overview of a curriculum, and we report on the more comprehensive document [33]. We excluded 199 full-text documents that did not meet the inclusion criteria, including five documents that we were unable to source an electric copy of [35-39], and two documents that were "working drafts" and subject to further revision $[40,41]$. We included one "work-

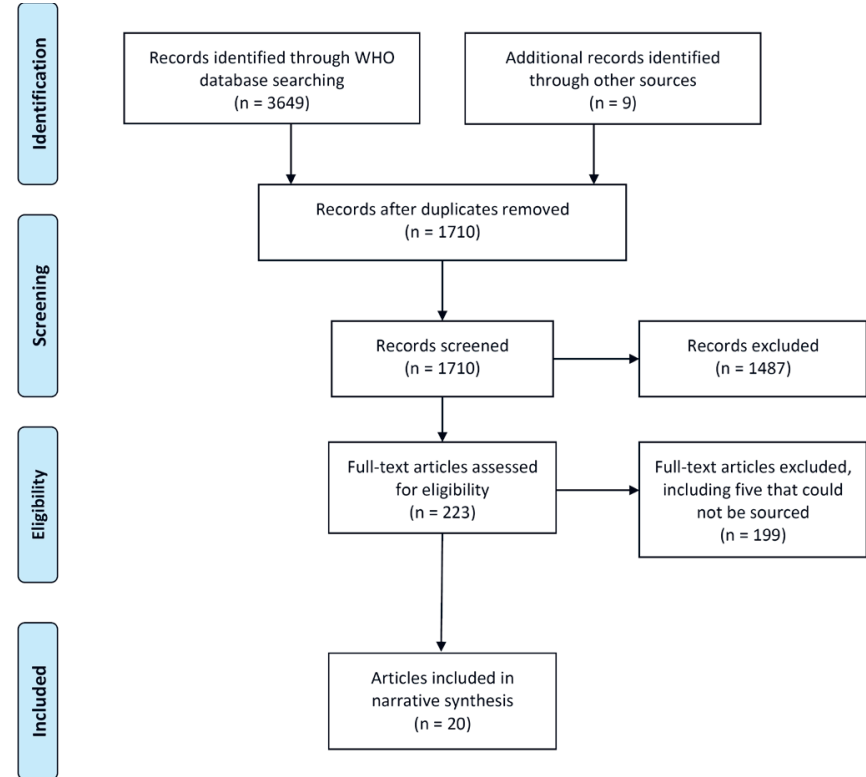

Figure 2. PRISMA flow diagram. ing draft" as it was recommended that the tool be made available to national programmes for immediate use [42].

\section{Global}

Of the 20 documents relevant to CDDs, 12 were global in scope. Six documents were about lymphatic filariasis (two described as "strategy", and the other four variously described as "aide-memoire", meeting report, handbook and manual; Table 1, column 1). The remaining six documents were about the PC-NTDS (described as guide, manual, practical advice, tool and training course (2)). Supportive evidence is provided in Table S2 in Online Supplementary Document.

Specifying aspects of the CDDs role: Of the 12 documents, seven made no reference to the responsibilities of CDDs in MDA (Table 1, column 3). Two training documents specified that CDDs have a role in drug delivery, stating that "CDDs play a critical role in distribution" and are responsible for leading "absentee and post-MDA follow-up". CDDs are also involved in distributing infor-

Table 1. Global documents.

\begin{tabular}{|c|c|c|c|c|c|c|}
\hline Column & 1 & 2 & 3 & 4 & 5 & 6 \\
\hline Year [ref] & Type of document* & $\begin{array}{l}\text { Disease (s) } \\
\text { covered }\end{array}$ & $\begin{array}{l}\text { Specifying aspects of the } \\
\text { CDDs role? }\end{array}$ & $\begin{array}{l}\text { Recommendations for } \\
\text { the CDDs role? }\end{array}$ & $\begin{array}{l}\text { Strategies for training } \\
\text { and managing CDDs? }\end{array}$ & $\begin{array}{l}\text { Other comments } \\
\text { about CDDs }\end{array}$ \\
\hline 2010 [43] & "Strategic plan" & LF & No & None & No & Included \\
\hline $2012[44]$ & "Provisional strategy" & $\mathrm{LF}^{*}$ & No & An option with caveats & Notes need & None \\
\hline $2013[45]$ & "Aide-memoire" & LF & No & None & Notes need & None \\
\hline $2016[46]$ & "Meeting report" & LF & No & None & Comment on aspects & None \\
\hline 2016b [47] & "Guide" & PC-NTDs & No & None & Comment on aspects & Included \\
\hline 2013b [48] & "Handbook" & LF & No & An option & Notes need & None \\
\hline $2010[49]$ & "Manual" & PC-NTDs & Data collection & None & Comment on aspects & Included \\
\hline $2011[50]$ & "Manual" & LF & Data collection & None & Comment on aspects & Included \\
\hline $2011[51]$ & "Practical advice" & PC-NTDs & Clinical care & None & No & Included \\
\hline $2016 c[42]$ & "Tool" & PC-NTDs & No & None & Comment on aspects & Included \\
\hline 2015 [52] & "Training course" & PC-NTDs ${ }^{8}$ & $\begin{array}{c}\text { Drug distribution; } \\
\text { data collection; health } \\
\text { promotion; clinical care }\end{array}$ & None & Comment on aspects & Included \\
\hline 2015b [53] & "Training course" & PC-NTDs & $\begin{array}{c}\text { Drug distribution; } \\
\text { data collection; health } \\
\text { promotion; clinical care }\end{array}$ & None & Comment on aspects & Included \\
\hline
\end{tabular}

CDD - community drug distributor, LF - lymphatic filariasis, PC-NTDs - preventive chemotherapy neglected tropical diseases, ref - reference

*Type of document is the words in the title that best classify the document.

†Comments about CDDs provided in the document's "background/introduction", or in a "progress report", an "update" or a "current situation", were not included as other comments about CDDs in policy.

¥Loiasis and malaria are also included in document.

§Also mentions foodborne trematodiases. 
mation, education and communication (IEC) materials and "other service delivery (MMDP)" (morbidity management and disability prevention). Some documents indicated that CDDs should carry out other tasks, including:

- collecting, recording and reporting information (four documents), such as completing PC tally sheet forms or registers, leading the community census and "monitoring inventory of logistics".

- health promotion (two documents), including leading community meetings, sensitisation and mobilisation, conducting IEC activities before and while distributing interventions, and lymphoedema/hydrocele case finding by going door-to-door to "disseminate the message".

- clinical management role (three documents), including assisting with adverse events (AEs) and referring people to a health facility, and record and report AEs. Actions for CDDs to manage AEs were provided, with a list of common AEs or symptoms, their frequency and treatment. There was an emphasis on drug safety (CDDs understanding treatment exclusion criteria, awareness of issues relating to treating children) and CDDs performing adequately ("CDDs must give the right information", "CDD ... and child should be relax, calm, friendly").

None made clear exactly what CDDs should do, or how they should do it.

Recommendations for the CDDs role: There were no recommendations for CDDs. Two documents for lymphatic filariasis mentioned CDDs may be of use to malaria programmes by encouraging net use.

Strategies for training and management: No document provided a comprehensive training and management strategy for CDDs. Two documents did not mention the topic, and three commented on the need for training and supervision but gave no further information. In the remaining seven, two training course documents provided some information of practical help for training. For example, "CDDs are community members who will be selected by the communities to undertake the task of drug distribution", the number of CDDs per hamlet to train (two), the gender ratio of CDD teams ("as much as possible choose a team of two people, a man and a woman") and length of training period (between two days). A list of what to include in CDD training was provided: "Diseases: Very brief description of diseases targeted, MDA strategy; Drugs: What drugs to use, Dosage, including use of dose poles; How to administer, Exclusion criteria, AEs/SAEs identification, management and referral; Register/Tally Sheets".

For CDD management, a training course included "Regular supervision and monitoring in the community", and the responsibilities of the different personnel overseeing CDDs was detailed. Some documents highlighted a need to inform community volunteers of the results of their work and discuss problems, including "drug administration, remuneration, compliance, IEC, form filling, use of remaining drugs...". Four documents included practical guidance for monitoring, with corrective actions to take with CDDs when interpreting and following up reported and surveyed coverage results, and in the event of Transmission Assessment Surveyl (TAS1) failure.

Other comments about CDDs: Scattered through the documents were statements that indirectly referred to the CDDs role and how they should be managed. This included benefits and challenges of integration for CDDs, such as "improved supervision of community drug distributors (CDDs)... with fewer resources" and "increasing demands are being placed on volunteers as other programmes recognize their potential". One document noted that "... for volunteers to deliver chemotherapy for a variety of NTDs and provide other health services, it will be necessary for health systems to create clear job descriptions, standardize incentives across programmes, and coordinate efforts to build capacity".

Other statements mostly centred around CDD performance, such as data quality concerns, operational errors and CDD attitude, and preparation for MDA, preparation for MDA, which consists of selection of CDDsCDDs (two people, a man and a woman, selected by the community or by district level personnel), mode of drug distribution (eg, house-to-house, from a central point, areas where people congregate or determined by the District NTD coordinator) and the MDA commodities required.

\section{Regional}

We identified eight regional documents. Five documents were about onchocerciasis (described as meeting reports (4) and handbook; Table 2, column 1), and one document was about onchocerciasis and lymphatic filariasis (described as "strategic plan of action"). The remaining two documents were about NTDs (described as "strategy" and meeting report). Supportive evidence is provided in Table S3 in the Online Supplementary Document. 
Table 2. Regional documents.

\begin{tabular}{|c|c|c|c|c|c|c|}
\hline Column & 1 & 2 & 3 & 4 & 5 & 6 \\
\hline Year [ref] & Type of document* & Disease $(\mathrm{s})$ covered & $\begin{array}{l}\text { Specifying aspects } \\
\text { of the CDDs role? }\end{array}$ & $\begin{array}{l}\text { Recommendations } \\
\text { for the CDDs role? }\end{array}$ & $\begin{array}{l}\text { Strategies for training } \\
\text { and managing CDDs? }\end{array}$ & $\begin{array}{l}\text { Other comments } \\
\text { about CDDs }\end{array}$ \\
\hline 2014 [26] & "Strategy" & NTDs & No & None & No & Included \\
\hline $2013[54]$ & $\begin{array}{l}\text { "Strategic plan of } \\
\text { action" }\end{array}$ & $\begin{array}{c}\text { LF and } \\
\text { onchocerciasis }\end{array}$ & No & None & Comment on aspects & Included \\
\hline 2007 [55] & "Meeting report" & Onchocerciasis & No & $\begin{array}{c}\text { Formal } \\
\text { recommendation }\end{array}$ & No & None \\
\hline 2008 [56] & "Meeting report" & Onchocerciasis & No & None & No & Included \\
\hline 2009 [57] & "Meeting report" & Onchocerciasis & No & None & No & Included \\
\hline $2012[58]$ & "Meeting report" & Onchocerciasis & No & None & Notes need & Included \\
\hline 2017 [59] & "Meeting report" & NTDs $^{\ddagger}$ & No & An option & No & None \\
\hline $\begin{array}{l}2012 b \\
{[33]}\end{array}$ & "Handbook" & Onchocerciasis $^{\S}$ & $\begin{array}{l}\text { Drug distribution; } \\
\text { data collection; } \\
\text { health promotion }\end{array}$ & An option & $\begin{array}{l}\text { Clearly and compre- } \\
\text { hensively outlined }\end{array}$ & Included \\
\hline
\end{tabular}

CDD - community drug distributor, LF - lymphatic filariasis, NTD - neglected tropical diseases, ref - reference

*Type of document is the words in the title that best classify the document.

$†$ Comments about CDDs provided in the document "background/introduction", or in a "progress report", an "update" or a "current situation", were not included as other comments about CDDs in policy.

$¥$ Addresses preventive chemotherapy NTDs and case management NTDs.

§Also mentions other diseases that may use community-directed interventions, such as lymphatic filariasis, schistosomiasis, trachoma and malaria (bed nets).

Specifying aspects of the CDDs role: Of the eight documents, seven made no reference to the role of CDDs (Table 2, column 3). One document, a trainers' handbook for a curriculum and training module on the CDI strategy for medical and nursing students, stated "Implementation of the intervention by community implementers" consists of "delivery of the interventions". Specific tasks that CDDs are responsible for performing included:

- "attend training and other educational programmes in respect of the intervention programme;

- provide information, education and communication to the community;

- conduct census and estimate the population that will need the service;

- collect or ensure that intervention commodities are available in the community;

- keep record of how the intervention was delivered;

- submit a record of intervention to the supply collection point;

- provide feedback to the community."

The document outlines the community's role in planning and decision-making for CDI, although given that the decisions about the drugs and the diseases targeted are already made, it is not clear what these decisions are really. There is almost no guidance for appropriate CDD workload and the number of interventions or other services volunteers can be responsible for.

Recommendations for the CDDs role: One of the eight documents made a recommendation about CDDs. The Joint Action Forum (JAF) for onchocerciasis control "strongly endorsed" a change in the ratio of CDDs to people treated, from one CDD for up to 250 people to one CDD for up to 100 people. One document provided an option for CDDs to carry out sensitisation on skin NTDs during MDA and one document listed several potential interventions for volunteer distribution, including maintain and distribute bed net supplies, and case management of malaria, pneumonia and diarrhoea.

Strategies for training and management: Five documents did not mention the topic, and one endorsed the need for training more women CDDs but no further information. One document provided a few vague options for managing CDDs dwindling motivation to work on a voluntary basis over an extended period.

One document outlined a comprehensive curriculum on the CDI strategy for students in medical and nursing schools. The guidance in this trainers' handbook included units on "setting up the CDI strategy", "supervision" and "monitoring and evaluation". Setting up the CDI strategy in a community was described in detail and included use of the APOC video for training CDDs. Instructions for conducting CDD training were not included but use of the original APOC training manual for CDDs was recommended, with audio visuals by NTD support initiatives where the training requires multiple inter- 
ventions, such as distributing bed nets and drugs for other NTDs. Two to three days should also be allocated for training CDDs.

For management, the CDI trainers' handbook included the frequency of supervision for CDDs (twice monthly), the supervisor role and tools, and examples of forms and checklists for supervision and monitoring (such as "Integrated supervision checklist for PHC programmes" and "Supervision checklist for CDTI"). Examples of problems that can be encountered during supervision and possible solutions/actions were provided, as well as examples of CDD supervision tasks.

Other comments about CDDs: Statements concerning CDDs were included in six of the eight documents. The trainers' handbook for CDI contained general statements throughout, including the community's role in incentivising CDDs, CDD selection (community informed of selection criteria and then community selects implementers) and modes of drug distribution (door-to-door, community visits volunteer's home, or at a central place). Two other documents included statements regarding incentives for CDDs, and one document acknowledged CDDs use in case finding for "eye care". The AFRO-regional strategy stated that "Cross-cutting interventions or activities... should be harmonized and streamlined...". Listed among the interventions and activities was "involvement of community volunteers or medicine distributors". For supporting morbidity management, one document specified that "involvement and collaboration of patients and their families, community volunteers and community health workers is essential".

\section{National programme master plans}

We obtained the current NTD master plan for Cameroon (2016-2020), Ghana (2016-2020), Liberia (2016-2020) and Nigeria (2015-2020). The Cameroon master plan is currently a draft and available in French only. This plan was translated to English, and we acknowledge that the plan may be less formulated in its' current format as a draft document. The plans range from 61 to 148 pages in length and consist of the main WHO recommended sections [27], including: situational analysis, NTD strategic agenda and operational framework, as well as budget justification and estimates (Figure 1). Our analysis is summarised in Table 3.

Anticipated role of CDDs: Each plan includes a table summarising the disease-specific goals and objectives, which generally indicates the main strategies/interventions, delivery channels and target population for each targeted disease. Community-based MDA is the strategy for onchocerciasis and lymphatic filariasis in all four plans, and for trachoma in Nigeria and Cameroon where the disease is endemic. All countries are endemic for schistosomiasis and STHs, and the strategy for schistosomiasis in the four plans and for STHs in three plans (excluding Ghana) consists of community-based targeted treatment to specific at-risk groups. Use of CDTI/CDI is indicated as the "delivery channel" in some of the strategies, but only the Liberia plan specifically mentions "community health volunteers" for STHs. Three of the plans (excluding Cameroon) provide a table detailing the packages of MDA, with "training community volunteers" as an overall requirement, implying their involvement in drug delivery. MDA is also included for yaws in two plans (Ghana and Liberia), but this requires health workers in Ghana and it is unclear whether community-wide treatment requires "staff" or volunteers in Liberia.

For case management and chronic care, two plans (Ghana and Liberia) include NTD volunteers. In Ghana, volunteers will conduct "Case search and management", and in Liberia, volunteers are implicated in routine active case detection and referral for 'case management' NTDs. For transmission control, two plans (Liberia and Nigeria) include volunteers. In Liberia, a community health volunteer (CHV) in each community will distribute insecticide treated nets (ITNs), and in Nigeria, community volunteers will be trained to use "LLINs, IRS and personal hygiene" (long lasting insecticidal nets, indoor residual spraying). The Liberia plan also includes health promotion, such as conducting meetings and providing kits and training to "affected individuals" to enable "home based self-care for CM-NTDs" (case management NTDs).

Two plans (Cameroon and Liberia) acknowledge other activities NTD volunteers have been involved with, including: delivering bed nets, vitamin A and immunisations, "family planning interventions ORS and zinc", identifying scrotal swelling and lymphedema cases, detection and referral of suspicious leprosy cases, social mobilisation, contact tracing, health education and "member of burial teams...during the EVD outbreak". The Nigeria plan also states that CDTI provided entry points for other community-based interventions.

Strategies for training and management: All the country master plans include aspects of training and supervision, but details on the frequency and individuals responsible for training and managing CDDs are not always described. 
Table 3. The role of community drug distributors in national neglected tropical diseases programme master plans for Cameroon, Ghana, Liberia and Nigeria*

\begin{tabular}{|c|c|c|c|c|c|c|}
\hline & \multicolumn{2}{|l|}{ National plan } & \multicolumn{4}{|c|}{ NTD PROGRAMME MASTER PLAN } \\
\hline & & Country & Cameroon & Ghana & Liberia & Nigeria \\
\hline & & Period & 2016-2020 & 2016-2020 & $2016-2020$ & 2015-2020 \\
\hline \multirow{10}{*}{$\begin{array}{l}\text { Anticipat- } \\
\text { ed role of } \\
\text { CDDs }\end{array}$} & NTD programme & Disease & & & & \\
\hline & \multirow[t]{6}{*}{ Drug distribution } & Onchocerciasis & MDA & MDA & MDA & MDA \\
\hline & & LF & MDA & MDA & MDA & MDA \\
\hline & & Schistosomiasis & Targeted & Targeted & Targeted & Targeted \\
\hline & & STHs & Targeted & Not required & Targeted & Targeted \\
\hline & & Trachoma & MDA & Not required & Not required & MDA \\
\hline & & Other NTDs & Not required & Not required & Unclear* & Not required \\
\hline & \multicolumn{2}{|c|}{ Case management and chronic care } & Not included & Yes & Yes & Not included \\
\hline & \multicolumn{2}{|c|}{ Transmission control (eg, bed net delivery) } & Not included & Not included & Yes & Yes \\
\hline & \multicolumn{2}{|c|}{ Other activities documented (eg, vitamin A distribution) } & Yes & Not included & Yes & Yes \\
\hline \multirow[t]{3}{*}{ Training } & \multicolumn{2}{|c|}{ Required by plan? } & Required & Required & Required & Required \\
\hline & \multicolumn{2}{|l|}{ Who will train? } & Not specified & "Personnel" & Not specified $\dagger$ & Not specified $\neq$ \\
\hline & \multicolumn{2}{|c|}{ Frequency of training } & Annually & Q2 and Q4 & Q1, Q2, Q3, Q4, p.a. & Annually§ \\
\hline \multirow[t]{3}{*}{ Supervision } & \multicolumn{2}{|l|}{ Required by plan? } & Required\| & Required & Required & Required \\
\hline & \multicolumn{2}{|c|}{ Who will supervise? } & Not specified & "Personnel" & Not specified & Not specified \\
\hline & \multicolumn{2}{|c|}{ Frequency of supervision } & Annually\| & $\begin{array}{l}\text { Q2, Q3, Q4. } \\
\text { Monthly sub-dis- } \\
\text { trict review meet- } \\
\text { ing (CDDs)gl }\end{array}$ & $\begin{array}{c}\text { Not specified. } \\
\text { Monthly meeting } \\
\text { with health facility } \\
\text { OIC (CHVs) }\end{array}$ & Not specified \\
\hline Incentives & \multicolumn{2}{|l|}{ Included in plan? } & Unclear** & Not included & Bicycles & $\begin{array}{l}\text { Usable IEC } \\
\text { materials }\end{array}$ \\
\hline
\end{tabular}

$\mathrm{CDD}$ - community drug distributor, CHV - community health volunteer, IEC - information, education and communication, LF - lymphatic filariasis, MDA - mass drug administration, NTD - neglected tropical diseases, OIC - Officer in Charge, p.a. - per annum, STH - soil-transmitted helminths. *We refer to "MDA" when mass drug administration is indicated, and "targeted" when treatment appears to be indicated for specific groups. We anticipated the distributors involvement when the drug delivery strategy included terms such as "community-based", "community directed intervention (CDI)", and "community directed treatment with ivermectin (CDTI)". The Liberia plan indicates community MDA for yaws but it is not clear whether this requires community volunteers or "staff".

$†$ The Liberia plan did not identify the trainer and supervisor of CDDs, although the "situation analysis" indicates this requires "health facility staff". For case management NTDs, cascade training will be used.

¥Training of CDDs for MDA is not described in the Nigeria plan. Training community implementers on the use of "LLINs, IRS and personal hygiene" requires training of health workers.

\$The Nigeria plan includes annual training of "community NTD implementers on the use of data collection reporting tools", but overall training for CDDs is not indicated.

"The Cameroon plan specifies "supervision of community-based actions".

ITThe Ghana plan states "conduct monitoring and supervision at all levels" for MDA. Sub-district monthly review meetings require "transport for CDDs". *** "Motivate community actors" annually is in Cameroon's plan but no further details.

For training, the Cameroon plan specifies annual training of "community agents" and "community actors in social mobilization", and community relay training in data management. For Ghana, "training of... community based volunteers will be done... jointly for all the diseases". CDDs will be trained to conduct MDA by "personnel" twice a year. The Liberia plan includes training of community volunteers "on integrated NTD activities" quarterly over 2017-2020, and training of a CHV in the third quarter annually to distribute bed nets. For case management, “... community volunteers (especially CDDs) shall be trained in the suspicion cases during CDI activities, and referral of suspected cases to the health facilities...", with integrated in-service cascade training of CHVs. For Nigeria, a priority is "comprehensive capacity building of NTD staff and volunteers at all levels on integrated NTDs". The plan includes annual training of community NTD implementers to use "data collection reporting tools", and on the use of "LLINs, IRS and personal hygiene" (requiring training of health workers to conduct training).

For supervision, the Cameroon plan includes "supervision of community-based actions" annually. In the Ghana plan, "personnel" and district staff are needed to monitor CDD training over 2013-2017. For MDA, "personnel" are required to "conduct monitoring and supervision at all levels" in periods "Q2, Q3, and Q4". Sub-district monthly reviews over 2013-2017 will also require "transport for CDDs". Supervision 
is listed as a requirement in the Liberia and Nigeria plans, and Liberia also plans to "integrate NTDs into monthly meeting of CHVs/CHA with health facility OIC to report on conditions within the community". However, no further details on CDD management are provided.

Motivation and incentives: As part of the situation analysis, all plans include a strengths, weaknesses, opportunities and threats (SWOT) analysis for the NTD programme. The plans all include the presence of "mobilizable", "committed" or "trained" CDDs in communities as a strength or opportunity, but also note challenges. The Cameroon and Ghana plans indicate lack of motivation as an issue, as well as the demand for incentives and ageing of the distributors. The Ghana and Liberia plans recognise that volunteers can be demotivated by other non-governmental organisation (NGO) activities and competing health activities and incentives between programmes. The Liberia plan states that "... lack of funding and incentives are an ongoing challenge for CDDs", but CDDs could be involved in income generating activities through collaboration with development NGOs and Agriculture Ministry. For the Nigeria NTD programme, high attrition, low commitment and inadequate numbers of trained community implementers are challenges.

For proposed activities, the Cameroon plan states "encourage communities to document motivation mechanisms of community workers", with development and dissemination of "motivation reporting tools for community workers". "Motivate community actors" annually is also part of the plan, but no details are provided. Incentives for CDDs in Liberia include "procure 3000 bicycles for CDDs" in the second quarter of 2017. In Nigeria, a priority is the "provision of incentives in form of usable IEC materials". The Ghana plan does not include incentives for CDDs.

\section{DISCUSSION}

The role of CDDs has changed over the years since APOC first started using the CDTI implementation model. With the intensified drive to meet current global health targets for NTDs controlled by drugs, delivery of PC by community volunteers appears critical for programme success but could potentially be a weak link [60-62]. This review consolidated the evidence from more recent WHO global and WHO-AFRO regional documents and select national NTD programme master plans in Africa. We found that the policies and plans assume CDDs will implement NTD programmes, but there was little delineation of responsibilities or up-to-date practical guidance. This may signal an absence of explicit policy and programme management, and this ambiguity probably impairs the effectiveness of these programmes.

Only three documents explicitly stated that CDDs will distribute drugs. Other tasks associated with drug distribution, such as health promotion, data collection and reporting, and clinical care, were also specified in few documents. These activities are fairly consistent with what was expected of CDDs under single-disease control guidance from APOC [1]. Additional activities identified in the more recent policy documents included "MMDP delivery", although what this constitutes was unclear. Some documents provided potential uses for CDDs, such as in delivery of bed nets and sensitisation for skin NTDs, and only one document from 2007 made a recommendation relating to CDDs [55]. Similarly, the role of CDDs in the national multi-year master plans was not explicit. For NTDs requiring drug distribution, the plans refer to community-based, use of CDTI/CDI structures, or MDA, with "volunteer training" listed as a requirement alongside these strategies. Half of the country master plans also involve volunteers in either case management or transmission control, and most acknowledge CDDs participation in other tasks outside control of PC-NTDs.

Training and management for CDDs was one of the most frequently addressed topics, particularly in global policy. Across the documents there was more detail on supervisory and management tasks compared to training. The more comprehensive of the WHO documents were the three training courses/modules $[33,52,53]$. Between them, aspects of training, MDA commodities, examples of supervision tasks, forms and recommended management styles were presented, but there was little in the way of practical guidance on how CDDs should conduct drug distribution and ancillary tasks (such as IEC) in integrated control programmes, let alone other service delivery. It is also not documented anywhere how many interventions CDDs can be responsible for. Training and supervision of CDDs was required by all the country master plans but reported variously. Training frequency ranged from annual in Nigeria and Cameroon to biannual in Ghana to quarterly in Liberia, and training was indicated for "integrated NTD activities" or "jointly for all the diseases" in some plans, and for specific functions such as "social mobilization" or "data collection reporting tools" in others. Details of CDD supervision and timing were less clearly formulated across the master plans. 
Sustainability of community-based programmes relies on a committed, motivated and sufficiently incentivised cadre of volunteers to deliver drug packages year after year and extend health interventions to communities underserved by current health systems. If the expectations are not clear, and if no one has responsibility to supervise them, then it is likely they will not be inspired to continue their voluntary work [63]. Incentives and CDD motivation were mentioned in some global and regional policy documents, and in all the country master plans; however, there was little consensus or solutions offered. For example, the governing body of APOC recommended developing a policy on incentives for volunteers at country levels and urged governments to address the incentive issues [56,57]. This was reiterated in the lymphatic filariasis strategic plan, which stated: "...for volunteers... it will be necessary for health systems to create clear job descriptions, standardize incentives across programmes...". However, other documents put the onus on the community to motivate volunteers [33]. The four country master plans all identified lack of motivation or high attrition of volunteers as threats or weaknesses to the NTD programme.

Poorly motivated volunteers and high attrition rates may be a consequence of the lack of clear guidance outlining the role of CDDs and how to train and supervise them, leading to uncertainty over responsibilities and how to perform tasks. This is a common challenge identified across empirical literature that explores the experiences of CDDs and evaluates their engagement with programme implementation. Improper training [64-66], insufficient supervision [67,68], confusion around responsibilities [69,70] and the limited amount of time allocated for workload $[66,68,71]$ have all been reported in NTD programme literature. Recent studies and literature reviews have also highlighted that factors influencing CDD motivation and performance include and extend beyond monetary and material incentives [72-74], although this is not unique to contemporary NTD volunteer schemes [75]. Intrinsic motivation, gender, cost to participate and health systems and community support were all identified as recurring themes in the literature [73]. Community support and the health system in particular were linked to motivation and performance, and in one study the CDDs who engaged friend groups with MDA were among the best performing CDDs [74]. Despite this evidence, and the possibly increased workload for CDDs through strategic scaling-up of national NTD programmes, most of the plans did not adequately address how volunteers will be incentivised or motivated.

This lack of policy and planning frequently results in a lack of regulations or strict obligation to support CDD volunteerism, which may negatively impact their motivation and retention. To date, challenges to CDD performance based on health systems factors outlined above have been considered at the implementation level. However, this review highlights that there are also clear gaps in policy and implementation guidelines regarding the most effective ways to train, supervise and support CDDs to fulfil their role to the best of their ability. These gaps seem to have become greater overtime, indicating a reliance on programme implementers to carry out business as usual in relation to the now historic CDTI strategy. CDDs are facing changing contexts, such as changes from rural distribution to growing urbanisation, fragile and conflict affected states, migration and nomadic communities, which also have CDDs. The role of CDDs has clearly evolved over the past two decades, but the changing nature of their role has not been reflected in policy and programme documentation. For example, a recent study of an integrated NTD programme in Uganda questioned some key assumptions of CDDs in community-based MDA, including the perceived altruism of volunteers (13.56\% were considered as altruistic) and the need to select volunteers through community-wide meetings (selection method had no association with treatment rates) [74]. The study demonstrated that volunteer characteristics and involvement of their friend groups may be a better way to select and monitor CDD performance [74]. This would suggest that NTD programmes need to work within existing health system infrastructure to strengthen the level of support provided to this critical cadre, whilst also considering how to document their role and management requirements clearly within programme guidelines. Agreeing the role of this cadre at the national level, as well as the development of clear training, supervision and management tools, could be a critical first step toward improving CDD performance in many settings.

Whilst our review of policy has focused on the African situation for CDDs in NTD programmes, the findings will likely apply to other global health programmes that rely on volunteers [76]. Given the diversity of health initiatives that rely on CHWs, who may also be unpaid volunteers not formally recognised by or integrated into health systems, and responsible for a myriad of tasks in the community, some ambiguity is to be expected. The recent publication of WHO guidelines on health policy and system support to optimise CHW programmes [77] is a step towards more formal recognition of their contributions in communities and the challenges faced by this cadre; however, our review has identified important issues in policy setting and programme planning that are probably reflected in other volunteer schemes operating worldwide. 
For this review, we attempted to identify WHO policies and plans that include CDDs as they are known by NTD programmes, which also involved searching broader CHW documents. Therefore, a key strength of this review is that it brings together all the CDD policy documents produced over the last ten years and indicates a key documentation gap, which is important in progressing NTD programmes. A potential weakness may lie in not reviewing national programme standard operating procedures (SOPs) and other policies that may impact on the CDDs role or management. For example, Nigeria's Federal Ministry of Health has produced documents such as the "training guide for trainers of community implementers (CIs) on NTD control and elimination", while in Liberia the role of CHWs and CDDs has become more formalised, where the programme is now encouraged not to use CDDs but to implement through the new CHW policy [78]. Our review has also given less attention to the role of CDDs within other NTD service delivery, such as integrated case management, as we did not find explicit evidence of their responsibilities in this beyond that which is documented in the national master plans. However, as global and regional policy and national master plans form the basis for NTD policy setting and programme implementation guidance, we feel this review highlights critical areas that need to be addressed.

\section{CONCLUSIONS}

Global and African WHO policy documents assume the implementation of CDDs but provide little guidance for their roles and how to prepare and utilise them within integrated programmes and changing contexts.

Given the changes in diseases covered, and adding of tasks such as morbidity control, case management, and distribution of other commodities, it seems likely the ambiguity and growth of tasks will, in the absence of clear guidance, regular training and active management at both national and local level, contribute to these programmes failing to assure equitable drug delivery to people.

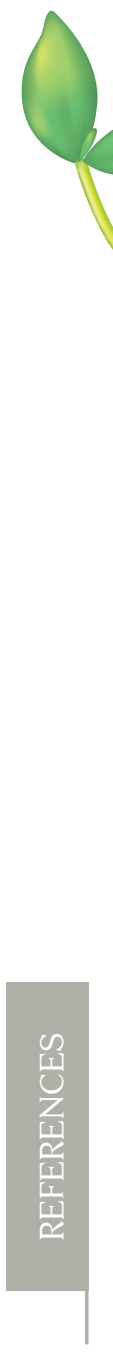

Acknowledgements: The authors would like to thank Dr Nicolas Pionnier for translating the Cameroon NTD master plan from French to English, and Dr Kim Ozano and Dr Luret Lar for comments on the final draft.

Funding: CM, LD and RT salaries are funded from the COUNTDOWN project, which is funded by the Department for International Development (DFID). PG also received funding from DFID to carry out this review and other relevant work.

Authorship contributions: All authors contributed to conception and design of the work. CM wrote the protocol with guidance from PG. CM designed the search strategy and conducted the search. CM conducted the analysis and prepared the manuscript with PG. All authors reviewed and provided input on later drafts of the manuscript. All authors read and approved the final manuscript and agreed to be accountable for all aspects of the work in ensuring that questions related to the accuracy or integrity of any part of the work are appropriately investigated and resolved.

Competing interests: CM, LD and RT are all funded by DFID-funded research project called COUNTDOWN, which aims to improve MDA programmes for NTDs. PG is co-ordinating editor of the Cochrane Infectious Diseases Group that carries out systematic reviews of the effects of interventions in NTDs. This analysis is an output of the 'evidence synthesis' theme of the project. The views expressed in the submitted article are the authors and not an official position of the funder or the COUNTDOWN consortium or DFID. All authors have completed the Unified Competing Interest form at www.icmje.org/coi_disclosure.pdf (available on request from the corresponding author), and declare no further conflicts of interest.

\section{Additional material}

Online Supplementary Document

1 African Programme for Onchocerciasis Control. Community directed treatment with ivermectin: a practical guide for trainers of community-directed distributors. Ouagadougou: African Programme for Onchocerciasis Control/World Health Organization; 1998.

2 World Health Organization. Crossing the billion. Preventive chemotherapy for neglected tropical diseases lymphatic filariasis, onchocerciasis, schistosomiasis, soil-transmitted helminthiases and trachoma. Gevena: World Health Organization; 2017.

3 World Health Organization. Report of the tenth meeting of the WHO strategic and technical advisory group for neglected tropical diseases: 29-30 March 2017 WHO, Geneva. Geneva: World Health Organization; 2017.

4 Hotez PJ, Fenwick A, Savioli L, Molyneux DH. Rescuing the bottom billion through control of neglected tropical diseases. Lancet. 2009;373:1570-5. Medline:19410718 doi:10.1016/S0140-6736(09)60233-6 
5 Litt E, Baker MC, Molyneux D. Neglected tropical diseases and mental health: a perspective on comorbidity. Trends Parasitol. 2012;28:195-201. Medline:22475459 doi:10.1016/j.pt.2012.03.001

6 Global Burden of Disease Study Collaborators. Global, regional, and national disability-adjusted life-years (DALYs) for 315 diseases and injuries and healthy life expectancy (HALE), 1990-2015: a systematic analysis for the Global Burden of Disease Study 2015. Lancet. 2016;388:1603-58. Medline:27733283 doi:10.1016/S0140-6736(16)31460-X

7 Hotez PJ. Stigma: the stealth weapon of the NTD. PLoS Negl Trop Dis. 2008;2:e230. Medline:18446206 doi:10.1371/ journal.pntd.0000230

8 Global Burden of Disease Study Collaborators. Global, regional, and national life expectancy, all-cause mortality, and cause-specific mortality for 249 causes of death, 1980-2015: a systematic analysis for the Global Burden of Disease Study 2015. Lancet. 2016;388:1459-544. Medline:27733281 doi:10.1016/S0140-6736(16)31012-1

9 World Health Organization. An African Region free of neglected tropical diseases: working group meeting on the establishment of a new NTD entity 28 - 30 April 2015, Johannesburg, South Africa: report. Available: https://apps.who.int/ iris/handle/10665/192602. Accessed: 30 April 2019.

10 World Health Organization. Preventive chemotherapy in human helminthiasis. coordinated use of anthelminthic drugs in control interventions: a manual for health professionals and programme managers. Geneva: World Health Organization; 2006.

11 World Health Organization. Community directed treatment with ivermectin: report of a multi-country study. Geneva: World Health Organization; 1996.

12 African Programme for Onchocerciasis Control. Empowering partnerships and communities: APOC and the fight to rid Africa of river blindness. Ouagadougou: African Programme for Onchocerciais Control; 2001.

13 Homeida M, Braide E, Elhassan E, Amazigo UV, Liese B, Benton B, et al. APOC's strategy of community-directed treatment with ivermectin (CDTI) and its potential for providing additional health services to the poorest populations. Ann Trop Med Parasitol. 2002;96 Supp 1:S93-S104. Medline:12081254 doi:10.1179/000349802125000673

14 Special Programme for Research and Training in Tropical Diseases. Community-directed treatment of lymphatic filariasis in Africa: report of a multi-country study in Ghana and Kenya. Geneva: World Health Organization; 2000.

15 World Health Organization. Prevention and control of schistosomiasis and soil-transmitted helminthiasis. First report of the Joint WHO Expert Committees, technical report series 912. Geneva: World Health Organization; 2002.

16 World Health Organization. Intensified control of neglected diseases: report of an international workshop, Berlin, 10-12 December 2003. Geneva: World Health Organization; 2004.

17 World Health Organization. Strategic and technical meeting on intensified control of neglected tropical diseases, a renewed effort to combat entrenched communicable diseases of the poor: report of an international workshop, Berlin, 1820 April 2005. Geneva: World Health Organization; 2006.

18 Molyneux DH, Hotez PJ, Fenwick A. "Rapid-impact interventions": how a policy of integrated control for Africa's neglected tropical diseases could benefit the poor. PLoS Med. 2005;2:e336. Medline:16212468 doi:10.1371/journal.pmed.0020336

19 Brady MA, Hooper PJ, Ottesen EA. Projected benefits from integrating NTD programs in sub-Saharan Africa. Trends Parasitol. 2006;22:285-91. Medline:16730230 doi:10.1016/j.pt.2006.05.007

20 Lammie PJ, Fenwick A, Utzinger J. A blueprint for success: integration of neglected tropical disease control programmes. Trends Parasitol. 2006;22:313-21. Medline:16713738 doi:10.1016/j.pt.2006.05.009

21 Hopkins DR, Eigege A, Miri ES, Gontor I, Ogah G, Umaru J, et al. Lymphatic filariasis elimination and schistosomiasis control in combination with onchocerciasis control in Nigeria. Am J Trop Med Hyg. 2002;67:266-72. Medline:12408665 doi:10.4269/ajtmh.2002.67.266

22 Hanson C, Weaver A, Zoerhoff KL, Kabore A, Linehan M, Doherty A, et al. Integrated implementation of programs targeting neglected tropical diseases through preventive chemotherapy: identifying best practices to roll out programs at national scale. Am J Trop Med Hyg. 2012;86:508-13. Medline:22403327 doi:10.4269/ajtmh.2012.11-1589

23 Neglected Tropical Disease Control Program. Neglected Tropical Disease Control Program: final report November 2012. Available: https://www.rti.org/sites/default/files/resources/ntdcp_final_report_lo_res.pdf. Accessed: 30 April 2019.

24 World Health Organization. Global plan to combat neglected tropical diseases 2008-2015. Geneva: World Health Organization; 2007.

25 London Declaration on Neglected Tropical Diseases. Uniting to combat NTDs. Available: http://unitingtocombatntds. org/sites/default/files/resource_file/london_declaration_on_ntds.pdf. Accessed: 30 April 2019.

26 World Health Organization Regional Office for Africa. Regional strategy on neglected tropical diseases in the WHO African Region 2014-2020. Brazzaville: World Health Organization Regional Office for Africa; 2014.

27 World Health Organization Regional Office for Africa. Guide for preparing a master plan for national neglected tropical diseases programmes in the African Region. Brazzaville: World Health Organization Regional Office for Africa; 2012.

28 World Health Organization. Report of the global partners' meeting on neglected tropical diseases. Geneva: World Health Organization; 2007.

29 Lewin S, Munabi-Babigumira S, Glenton C, Daniels K, Bosch-Capblanch X, van Wyk BE, et al. Lay health workers in primary and community health care for maternal and child health and the management of infectious diseases. Cochrane Database Syst Rev. 2010;(3):CD004015. Medline:20238326 doi:10.1002/14651858.CD004015.pub3

30 Regional Committe for Africa 63. Regional strategic plan for neglected tropical diseases in the African Region 2014-2020. Brazzaville: World Health Organization Regional Office for Africa; 2013.

31 Regional Committe for Africa 63. Regional strategy on neglected tropical diseases in the WHO African Region (Document AFR/RC63/10). Brazzaville: World Health Organization Regional Office for Africa; 2013. 
32 Regional Committe for Africa 63. Regional strategy on neglected tropical diseases in the WHO African Region $2014-$ 2020. Brazzaville: World Health Organization Regional Office for Africa; 2013.

33 African Programme for Onchocerciasis Control. Curriculum and training module on the community-directed intervention (CDI) strategy for faculties of medicine and health sciences - trainers' handbook. Ouagadougou: African Programme for Onchocerciasis Control/World Health Organization; 2012.

34 African Programme for Onchocercias Control. Curriculum and training module on the community-directed intervention (CDI) strategy for faculties of medicine and health sciences (2nd edition). Ouagadougou: African Programme for Onchocerciasis Control/World Health Organization; 2012.

35 African Programme for Onchocerciasis Control. Report of the thirty-fifth session of the Technical Consultative Committee (TCC), Ouagadougou 10-14 September 2012. Available: http://www.who.int/apoc/about/structure/tcc/en/.Accessed: 30 April 2019.

36 World Health Organization. Curriculum and training module on the community-directed intervention (CDI) strategy for faculties of medicine and health sciences. Geneva: World Health Organization; 2009.

37 World Health Organization Regional Office for Africa. Regional programme meeting; forty-sixth session (RPM 46): final report Geneva; Switzerland; 3-5 November 2011. Brazzaville: World Health Organization Regional Office for Africa; 2011.

38 World Health Organization Regional Office for Africa. Report on the first meeting on preventive therapy; June 30-July 04; 2014 (including a report of the RPRG LF sub-committee meeting held on April 23-25 2014). Brazzaville: World Health Organizarion Regional Office for Africa; 2014.

39 World Health Organization Regional Office for Africa. Towards accelerated reduction of neglected tropical diseases: WHO joint strategic plan for control of neglected tropical diseases; 2010-2015. Brazzaville: World Health Organization Regional Office for Africa; 2012.

40 World Health Organization. Towards universal coverage for preventive chemotherapy for neglected tropical diseases: guidance for assessing "who is being left behind and why": working draft for further piloting during 2018-2019. Geneva: World Health Organization; 2017.

41 ENVISION. Data quality assessment for neglected tropical diseases: guidelines for implementation, December 2013, working draft for field-testing. Available: https://www.ntdenvision.org/resource/publication/data_quality_assessment_ dqa_for_ntds. Accessed : 30 April 2019.

42 World Health Organization. Rapid monitoring of preventive chemotherapy coverage in neglected tropical disease programmes: supervisor's coverage tool. Geneva: World Health Organization; 2016.

43 World Health Organization. Lymphatic filariasis: progress report 2000-2009 and strategic plan 2010-2020. Geneva: World Health Organization; 2010.

44 World Health Organization. Provisional strategy for interrupting lymphatic filariasis transmission in loiasis-endemic countries: report of the meeting lymphatic filariasis, malaria and integrated vector management, Accra, Ghana, 5-9 March 2012. Geneva: World Health Organization; 2012.

45 World Health Organization. Lymphatic filariasis: managing morbidity and preventing disability - an aide-memoire for national programme managers. Geneva: World Health Organization; 2013.

46 World Health Organization. Responding to failed transmission assessment surveys. Report of an ad hoc meeting: strategic and technical advisory group for neglected tropical diseases subgroup on disease-specific indicators, 4 December 2015, Washington (DC), USA. Geneva: World Health Organization; 2016.

47 World Health Organization. Coverage evaluation surveys for preventive chemotherapy: field guide for implementation. Geneva: World Health Organization; 2016.

48 World Health Organization. Lymphatic filariasis: practical entomology - a handbook for national elimination programmes. Geneva: World Health Organization; 2013.

49 World Health Organization. Monitoring drug coverage for preventive chemotherapy. Geneva: World Health Organization; 2010

50 World Health Organization. Lymphatic filariasis: monitoring and epidemiological assessment of mass drug administration - a manual for national elimination programmes. Geneva: World Health Organization; 2011.

51 World Health Organization. Assuring safety of preventive chemotherapy interventions for the control of neglected tropical diseases: practical advice for national programme managers on the prevention, detection and management of serious adverse events. Geneva: World Health Organization; 2011.

52 World Health Organization. Programme managers' training course for NTDs targeted for control or elimination by preventive chemotherapy interventions. Available: http://www.who.int/neglected_diseases/training/programme_managers/ en/. Accessed: 30 April 2019.

53 World Health Organization. District level management NTD training course. Available: http://www.who.int/neglected_ diseases/training/modules_for_district_level_management/en/. Accessed: 30 April 2019.

54 African Programme for Onchocerciasis Control-World Health Organization. Programme for the Elimination of Neglected Diseases in Africa (PENDA): strategic plan of action and indicative budget 2016-2025. Ouagadougou: African Programme for Onchocerciasis Control/World Health Organization; 2013.

55 African Programme for Onchocerciasis Control. Thirteenth session of the Joint Action Forum, Brussels, Belgium, 4-7 December 2007 - final communique. Available: http://www.who.int/apoc/about/structure/jaf/en/. Accessed: 30 April 2019.

56 African Programme for Onchocerciasis Control. Fourteenth session of the Joint Action Forum, Kampala, Uganda, 8-11 December 2008 - final communique. Available: http://www.who.int/apoc/about/structure/jaf/en/. Accessed: 30 April 2019.

57 African Programme for Onchocerciasis Control. Fifteenth session of the Joint Action Forum, Tunis, Tunisia, 8-10 December 2009. Available: http://www.who.int/apoc/about/structure/jaf/en/. Accessed:30 April 2019. 
58 African Programme for Onchocerciasis Control. Eighteenth session of the Joint Action Forum, Bujumbura, Burundi, 11-13 December 2012 - final communique. Available: http://www.who.int/apoc/about/structure/jaf/en/. Accessed: 30 April 2019.

59 World Health Organization Regional Office for Africa. Summary report, action points and recommendations of the first joint meeting on preventive chemotherapy and case management NTDs, Libreville, Gabon, 20-22 June 2017. Available: http://espen.afro.who.int/system/files/content/resources/Summary_And_Recommendations_of_JointPC_AndCM_NTD_ Prog_Mgrs_Meeting_June2017_English.pdf. Accessed: 30 April 2019.

60 Parker M, Allen T. Does mass drug administration for the integrated treatment of neglected tropical diseases really work? Assessing evidence for the control of schistosomiasis and soil-transmitted helminths in Uganda. Health Res Policy Syst. 2011;9:3. Medline:21211001 doi:10.1186/1478-4505-9-3

61 Kolaczinski JH, Kabatereine NB, Onapa AW, Ndyomugyenyi R, Kakembo AS, Brooker S. Neglected tropical diseases in Uganda: the prospect and challenge of integrated control. Trends Parasitol. 2007;23:485-93. Medline:17826335 doi:10.1016/j.pt.2007.08.007

62 Kabatereine NB, Malecela M, Lado M, Zaramba S, Amiel O, Kolaczinski JH. How to (or not to) integrate vertical programmes for the control of major neglected tropical diseases in sub-Saharan Africa. PLoS Negl Trop Dis. 2010;4:e755. Medline:20614017 doi:10.1371/journal.pntd.0000755

63 Walt G, Gilson L, Heggenhougen K, Knudsen T, Owuor-Omondi L, Perera M. Community health workers in national programmes: just another pair of hands? Buckingham: Open University Press Philadelphia; 1990.

64 Njomo DW, Amuyunzu-nyamongo M, Magambo JK, Njenga SM. The role of personal opinions and experiences in compliance with mass drug administration for lymphatic filariasis elimination in Kenya. PLoS One. 2012;7:e48395. Medline:23185256 doi:10.1371/journal.pone.0048395

65 York KJ, Kabole I, Mrisho M, Berry DM, Schmidt E. Factors affecting community participation in the CDTI program in Morogoro, Tanzania. J Nurs Scholarsh. 2015;47:96-104. Medline:25565278 doi:10.1111/jnu.12121

66 Kisoka W, Mushi D, Meyrowitsch D, Malecela M, Simonsen PE, Tersbøl BP. Dilemmas of community-directed mass drug administration for lymphatic filariasis control: a qualitative study from urban and rural Tanzania. J Biosoc Sci. 2017;49:447-62. Medline:27470198 doi:10.1017/S0021932016000365

67 Senyonjo L, Oye J, Bakajika D, Biholong B, Tekle A, Boakye D, et al. Factors associated with ivermectin non-compliance and its potential role in sustaining Onchocerca volvulus transmission in the west region of Cameroon. PLoS Negl Trop Dis. 2016;10: e0004905. Medline:27529622 doi:10.1371/journal.pntd.0004905

68 Njomo DWA-NM, Magambo JK, Ngure PK, Njenga SM. Factors associated with the motivation of community drug distributors in the Lymphatic Filariasis Elimination Programme in Kenya. S Afr J Infect Dis. 2012;27:66-70. doi:10.1080/ 10158782.2012 .11441487

69 Chami GF, Kontoleon AA, Bulte E, Fenwick A, Kabatereine NB, Tukahebwa EM, et al. Diffusion of treatment in social networks and mass drug administration. Nat Commun. 2017;8:1929. Medline:29208898 doi:10.1038/s41467-017-01499-z

70 Omedo MO, Matey EJ, Awiti A, Ogutu M, Alaii J, Karanja DMS, et al. Community health workers' experiences and perspectives on mass drug administration for schistosomiasis control in Western Kenya: the SCORE project. Am J Trop Med Hyg. 2012;87:1065-72. Medline:23091190 doi:10.4269/ajtmh.2012.12-0435

71 da-Costa Vroom FBAR, Anto F, Aikins M, Gyapong M, Gyapong J. Data reporting constraints for the lymphatic filariasis mass drug administration activities in two districts in Ghana: a qualitative study. SAGE Open Med. 2015;3: 2050312115594083. Medline:26770791 doi:10.1177/2050312115594083

72 Fleming FM, Matovu F, Hansen KS, Webster JP. A mixed methods approach to evaluating community drug distributor performance in the control of neglected tropical diseases. Parasit Vectors. 2016;9:345. Medline:27305942 doi:10.1186/ s13071-016-1606-2

73 Krentel A, Gyapong M, Mallya S, Boadu NY, Amuyunzu-Nyamongo M, Stephens M, et al. Review of the factors influencing the motivation of community drug distributors towards the control and elimination of neglected tropical diseases (NTDs). PLoS Neg Trop Dis. 2017;11:e0006065. Medline:29211746 doi:10.1371/journal.pntd.0006065

74 Chami GF, Kabatereine NB, Tukahebwa EM. Profiling the best-performing community medicine distributors for mass drug administration: a comprehensive, data-driven analysis of treatment for schistosomiasis, lymphatic filariasis, and soil-transmitted helminths in Uganda. BMC Med. 2019;17:69. Medline:30917824 doi:10.1186/s12916-019-1303-z

75 Karabi B, Peter W, LeBan Karen MT. Community health worker incentives and disincentives: how they affect motivation, retention, and sustainability. Arlington, Virginia: Basic Support for Institutionalizing Child Survival Project (BASICS II); 2001.

76 Kok MC, Broerse JE, Theobald S, Ormel H, Dieleman M, Taegtmeyer M. Performance of community health workers: situating their intermediary position within complex adaptive health systems. Hum Resour Health. 2017;15:59. Medline:28865471 doi:10.1186/s12960-017-0234-z

77 World Health Organization. WHO guideline on health policy and system support to optimize community health worker programmes. Geneva: World Health Organization; 2018.

78 Liberia Ministry of Health and Social Welfare. National policy and strategy on community health services. Available: http:// liberiamohsw.org/Policies\%20\&\%20Plans/Community\%20Health\%20Services\%20Policy.pdf. Accessed: 30 April 2019. 